\title{
Moderate hyperuricemia ameliorated kidney damage in a low-renin model of experimental renal insufficiency
}

Venla Kurra ( $\sim$ venla.kurra@tuni.fi )

Tampere University

Arttu Eräranta

Tampere University

Timo Paavonen

Tampere University

Teemu Honkanen

Tampere University

Juhani Myllymäki

Tampere University

\section{Asko Riutta}

Tampere University

Ilkka Tikkanen

Minerva Institute for Medical Research

Päivi Lakkisto

Minerva Institute for Medical Research

Jukka Mustonen

Tampere University

Ilkka Pörsti

Tampere University

\section{Research Article}

Keywords: experimental renal insufficiency, hyperuricemia, kidney morphology, oxonic acid

Posted Date: February 21st, 2022

DOI: https://doi.org/10.21203/rs.3.rs-1320919/v1

License: (c) (i) This work is licensed under a Creative Commons Attribution 4.0 International License.

Read Full License 


\section{Abstract}

Background: Hyperuricemia may predispose to renal damage, but in end-stage renal disease lower uric acid concentrations have been associated with higher mortality. In experimental studies, uric acid has promoted renal fibrosis and inflammation, but some studies have shown nephroprotective effects probably due to alleviated oxidative stress. We studied the influence of moderate hyperuricemia on kidney morphology in 5/6 nephrectomized rats.

Methods: Three weeks after subtotal nephrectomy or sham-operation, rats were put on $2.0 \%$ oxonic acid (uricase inhibitor) diet for 9 weeks. Blood pressure was monitored using tail-cuff. At close of the study, blood, urine, and kidney samples were taken, and renal histology, mast cell count, and oxidative stress markers were determined. Kidney tissue inflammation and fibrosis were evaluated using RT-PCR and immunohistochemistry.

Results: Oxonic acid diet increased plasma uric acid levels by $>80 \mu \mathrm{mol} / \mathrm{I}$ without influencing blood pressure. Creatinine clearance was reduced by $~ 60 \%$ in both remnant kidney groups, and by $30 \%$ in hyperuricemic sham-operated rats. In remnant kidney rats with suppressed plasma renin activity, moderate hyperuricemia decreased glomerulosclerosis, tubulointerstitial damage, and kidney mast cell count, but did not influence the fibrosis marker collagen I mRNA content. In both hyperuricemic groups, the mast-cell product 11-epi-prostaglandin- $\mathrm{F}_{2 a}$ excretion to the urine and kidney tissue COX-2 levels were decreased.

Conclusions: Hyperuricemic remnant kidney rats displayed improved kidney morphology and reduced markers of oxidative stress and inflammation. Thus, moderately increased plasma uric acid had beneficial effects on the kidney in this low-renin model of experimental chronic renal insufficiency.

\section{Introduction}

Hyperuricemia is a common finding in chronic renal insufficiency (CRI), mainly due to decreased renal uric acid (UA) excretion caused by reduced glomerular filtration and tubulointerstitial damage (1). Hyperuricemia may be injurious to the kidneys and the cardiovascular system, but whether hyperuricemia is a culprit or merely a marker of renal injury remains a matter of debate (2).

Experimental and clinical studies have linked hyperuricemia with enhanced cardiovascular complications and progression of CRI. In animal models, oxonic acid (OxO) induced hyperuricemia has been associated with stimulation of the renin-angiotensin system (RAS), endothelial dysfunction, oxidative stress, and salt-sensitive hypertension (3-6). In patients with chronic kidney disease, hyperuricemia correlated with the severity of glomerulosclerosis and interstitial fibrosis in renal biopsy $(7,8)$. In contrast, rats receiving intraperitoneal UA infusions presented with antihypertensive and nephroprotective effects, probably due to the antioxidant effects of UA (9-11). A protective role for UA during acute ischemic stroke has also been reported (12-15). 
In the absence of previous renal impairment, harmful hyperuricemia-associated morphological changes have been reported in rat kidneys $(5,16-18)$. A diet with $2 \%$ of the uricase inhibitor oxonic acid was found to increase collagen deposition, macrophage infiltration, juxtaglomerular renin staining, and medialumen ratio of afferent arterioles, and induce glomerular hypertrophy in rat kidneys (16-18). When Oxo was given daily by gastric gavage to rats for five weeks, afferent arteriolopathy and signs of increased intrarenal oxidative stress were observed (5). In experimental models of CRI, two reports associated hyperuricemia with harmful effects on the kidneys. In rats subjected to surgical 5/6 nephrectomy (NX), $2 \%$ Oxo diet for 6 weeks induced afferent arteriolopathy, enhanced glomerulosclerosis and interstitial fibrosis, and increased cyclooxygenase-2 (COX-2) and renin expression in preglomerular vessels (6). In the ligation type of $5 / 6 \mathrm{NX}$, which is a high-renin model of CRI, daily administration of Oxo by gastric gavage for 5 weeks induced renal cortical vasoconstriction and afferent arteriole thickening (19).

Previously, we found both harmful (20) and beneficial effects (9) in response to hyperuricemia induced by $2.0 \%$ Oxo feeding in rats subjected to surgical $5 / 6 \mathrm{NX}$, which is a low-renin model of CRI (20).

Hyperuricemia increased circulating RAS activity and promoted urinary $\mathrm{K}^{+}$loss (20), but also reduced oxidative stress in vivo as shown by increased plasma antioxidant capacity and decreased urinary 8isoprostaglandinF $\mathrm{F}_{2 a}$ excretion, and improved nitric oxide (NO) mediated vasorelaxation in the carotid artery (9).

In the present study, the hypothesis whether moderate experimental hyperuricemia influences kidney structure was further tested. We determined renal histology and markers of inflammation and fibrosis in 5/6 NX and Sham-operated rats that ingested 2.0\% Oxo diet for 9 weeks. Before the diet, our protocol included a 3-week recovery period after the NX surgery to reduce potential selection bias. In the remnant kidney model of CRI, experimental hyperuricemia improved kidney morphology, reduced oxidative stress, and decreased markers of inflammation.

\section{Methods}

\subsection{Animals and experimental design}

Male Sprague-Dawley rats were housed in an animal laboratory with free access to water and chow (Lactamin R34, AnalyCen, Lindköping, Sweden) containing $0.9 \%$ calcium, $0.8 \%$ phosphorus, $0.27 \%$ sodium, $0.2 \%$ magnesium, $0.6 \%$ potassium, $16.5 \%$ protein, $4.0 \%$ fat, $58 \%$ nitrogen-free extract, $3.5 \%$ fiber, $6.0 \%$ ash, $10 \%$ water, and $12550 \mathrm{~kJ} / \mathrm{kg}$ energy. At the age of 8 weeks (study week 0 ), the rats were anesthetized with ketamine (Parke-Davis Scandinavia AB, Solna, Sweden) plus diazepam (Orion Pharma Ltd., Espoo, Finland) using intraperitoneal doses 75 and $2.5 \mathrm{mg} / \mathrm{kg}$, respectively, and $N X(n=24)$ was carried out by the removal of upper and lower poles of the left kidney and the whole right kidney. The kidneys of the Sham rats $(n=24)$ were decapsulated. Anesthesia, antibiotics and treatment of postoperative pain were as previously reported $(21,22)$. 
Three weeks after the operations rats were assigned to 4 groups ( $n=12$ in each): Sham, Sham-Oxo, NX and NX-Oxo. The groups were formed so that mean systolic blood pressures (BP) and body weights in the Sham and Sham + Oxo, and NX and NX + Oxo groups, respectively, were similar. The $2.0 \%$ Oxo (SigmaAldrich Chemical Co, St. Louis, MO, USA) diet was fed to Sham-Oxo and NX-Oxo for 9 weeks, while the Sham and NX groups continued normal diet. Systolic BP was measured at $28^{\circ} \mathrm{C}$ by the tail-cuff method as the averages of five recordings in each rat (Model 129 BP Meter; IITC Inc., Woodland Hills, CA, USA). The 24-hour urine output was collected in metabolic cages at study weeks 3 and 12 and urine samples stored at $-80^{\circ} \mathrm{C}$ until analyses.

After 9 weeks of the Oxo diet the rats were anesthetized with urethane $(1.3 \mathrm{~g} / \mathrm{kg})$ and blood samples from cannulated carotid artery were drawn into chilled tubes with heparin or EDTA as anticoagulants. The kidneys were harvested, kidney halves were frozen in isopentane at $40^{\circ} \mathrm{C}$ and stored at $80^{\circ} \mathrm{C}$. The other kidney halves were fixed in $4 \%$ formaldehyde for 24 hours and embedded in paraffin. The study was approved by the Animal Experimentation Committee of the University of Tampere, and the Provincial Government of Western Finland Department of Social Affairs and Health, Finland (decision LSLH-20039718/Ym-23), and conforms to the Guiding Principles for Research Involving Animals.

\subsection{Blood and plasma determinations}

Plasma creatinine was measured using a standard clinical chemical method (Cobas Integra 800 Clinical Chemical Analyzer, Roche Diagnostics, Basel, Switzerland). UA was measured using an enzymatic colorimetric method (23) and plasma renin activity using a GammaCoat assay (Diasorin SpA Saluggia, Italy). All other determinations were carried out as described earlier $(21,22)$.

\subsection{Kidney morphology and immunohistochemistry}

Five micrometer-thick kidney sections were stained with hematoxylin-eosin, periodic acid Schiff (PAS), toluidine blue, or immunohistochemistry, and processed for light microscopic evaluation. All microscopic analyses were performed by an expert blinded to the treatments.

\subsubsection{Glomerulosclerosis (hematoxylin-eosin and PAS stain)}

One hundred glomeruli from each rat were examined at a magnification of $\times 400$ and scored from 0 to 5 (24): 0 = normal, 1 = mesangial expansion or basement membrane thickening; 2 = segmental sclerosis in $<25 \%$ of the tuft, $3=$ segmental sclerosis in $25-50 \%$ of the tuft, $4=$ diffuse sclerosis in $>50 \%$ of the tuft, 5 $=$ diffuse glomerulosclerosis, tuft obliteration and collapse. The damage index for each rat was calculated as a mean of the scores.

\subsubsection{Tubulointerstitial damage (hematoxylin-eosin and PAS stain)}


Injury consisting of tubular atrophy, dilatation, casts, interstitial inflammation, and fibrosis was assessed in 10 kidney fields at a magnification of $\times 100$ (24). Damage scoring was performed from 0 to 4: $0=$ normal, $1=$ lesions $<25 \%$ of the area, $2=$ lesions in $25-50 \%$ of the area, $3=$ lesions in $>50 \%$ of the area, 4 $=$ lesions covering the whole area.

\subsubsection{Arteriosclerosis index (PAS-stain)}

Small arteries were identified from kidney samples magnified $\times 400$, and graded from 0 to 2: $0=$ no hyaline thickening, $1=$ mild to moderate hyaline thickening in at least one arteriole, $2=$ moderate or severe hyaline thickening in more than one arteriole (25).

\subsubsection{Kidney mast cells (toluidine blue-stain)}

Toluidine blue staining was applied for mast cell identification and quantification. The number of the purple-stained mast cells were counted at a magnification of $\times 400$ and related to kidney tissue area.

\subsubsection{Immunohistochemistry COX-2, and smooth muscle cell alpha-actin (SMA)}

For the staining of COX-2 a 1:200 dilution of monoclonal anti-COX-2 IgG antibody (clone 33, BD Biosciences, San Diego, CA, USA) and for SMA a 1:200 dilution of monoclonal anti-SMA IgG antibody (code M0851, Dako Denmark A/S, Glostrup, Denmark) were used. Immunostaining was performed using the Ventana BenchMark LT Automated IHC Stainer (Ventana Medical System, AZ, USA) with the Ultraview Universal DAB detection kit (catalog no. 760 - 500, Ventana Medical System) as previously described (26). Tubulointerstitial COX-2 staining was scored 0 to $3: 0=$ no cells stained, $1=$ faint immunoreactivity, 2 = moderate positive staining, 3 = strong positive staining. Cell positivity (percentage of positive cells) was defined: $0=$ no cells stained, $1=1-25 \%$ positive cells, $2=26-75 \%$ positive cells, and $3=>75 \%$ positive cells. The results of both analyses were combined for the final score. In the glomeruli, the numbers of COX2 positive cells were counted and related to tissue area. Staining of SMA was evaluated in an attempt to identify afferent arterioles from the efferent arterioles (17).

\subsection{Kidney heme oxygenase-1 (HO-1) and collagen I mRNA with real-time quantitative PCR}

Total RNA was isolated from rat kidney tissue using Trizol reagent (Invitrogen, Carlsbad, CA, USA) and reverse transcription of RNA was performed using M-MLV reverse transcriptase (Invitrogen). The expressions of collagen-I and HO-1 mRNAs were studied using real-time quantitative RT-PCR. PCR reactions were performed with SYBR Green chemistry using ABI PRISM 7000 sequence detection system (Applied Biosystems, Foster City, CA, USA). PCR reactions for collagen I and H01 were performed in duplicate in a $25 \mu \mathrm{l}$ final volume containing 1 X SYBR Green Master mix (Applied Biosystems) and $300 \mathrm{nM}$ of primers. PCR cycling conditions were $10 \mathrm{~min}$ at $95^{\circ} \mathrm{C}$ and 40 cycles of 20 seconds at $95^{\circ} \mathrm{C}$ and $1 \mathrm{~min}$ at $60^{\circ} \mathrm{C}$. Data were analyzed using the absolute standard curve method and the amplification of a 
housekeeping gene $18 \mathrm{~S}$ was used for normalizing the results. The unnormalized expression of the control housekeeping gene 18S mRNA did not differ between the experimental groups (data not shown).

\subsection{Urine 11-epi-prostaglandin- $\mathrm{F}_{2 a}$}

The concentration of 11-epi-prostaglandin- $\mathrm{F}_{2 \mathrm{a}}$ was determined from urine collected in metabolic cages after selective solid-phase extraction by radioimmunoassay as previously described (27).

\subsection{Data presentation and analysis of results}

For normally distributed variables, statistical analyses were carried out using one-way and two-way analyses of variance (ANOVA), as appropriate. For variables with skewed distribution, the Kruskal-Wallis test was applied, with the Mann-Whitney U-test in the post-hoc analyses. Spearman's two-tailed correlation coefficients $\left(r_{S}\right)$ were calculated. Differences between the groups were considered significant when $P<0.05$, and the Bonferroni correction was applied in the post-hoc analyses. The results were expressed as means and standard errors of the mean (SEM), or as medians, 25th to 75th percentiles, and ranges. Unless otherwise indicated the $P$ values in the text refer to one-way ANOVA. The statistics were performed using IBM SPSS version 26.0 (Armonk, New York, USA).

\section{Results}

\subsection{Animal data}

In the beginning of the $2 \%$ Oxo diet (study week 3 ), body weights and systolic BPs were similar in the study groups (Table 1). At study week 12, however, body weights were lower in the NX-Oxo group versus NX rats, and two-way ANOVA analysis showed a significant lowering effect of $2 \%$ Oxo feeding on body weight $(p=0.004)$. During the follow up, the two NX rat groups displayed a modest elevation of BP when compared with the two Sham groups (two-way ANOVA $p=0.041$ ). Urine outputs were higher in both NX groups at study weeks 3 and 12 than in the Sham groups. The body weight-adjusted total kidney tissue weight was lower in the NX groups than in the Sham groups, but the remnant left kidney weight in the NX rats was higher than the left kidney weight in the Sham rats (Table 1). 
Table 1

Experimental group data and laboratory findings at study weeks 3 and 12 .

\begin{tabular}{|c|c|c|c|c|}
\hline & $\begin{array}{l}\text { Sham } \\
(n=9-12)\end{array}$ & $\begin{array}{l}\text { Sham + Oxo } \\
(n=12)\end{array}$ & $\begin{array}{l}N X \\
(n=11-12)\end{array}$ & $\begin{array}{l}N X+O \times O \\
(n=12)\end{array}$ \\
\hline \multicolumn{5}{|l|}{ Body weight (g) } \\
\hline Week 3 & $339 \pm 6$ & $338 \pm 7$ & $333 \pm 8$ & $332 \pm 7$ \\
\hline Week 12 & $433 \pm 8$ & $412 \pm 11^{\#}$ & $448 \pm 10$ & $411 \pm 8^{\dagger \#}$ \\
\hline \multicolumn{5}{|l|}{ Systolic blood pressure $(\mathrm{mmHg})$} \\
\hline Week 3 & $120 \pm 4$ & $121 \pm 5$ & $127 \pm 5$ & $125 \pm 5$ \\
\hline Week 12 & $134 \pm 7$ & $136 \pm 5$ & $142 \pm 6^{\ddagger}$ & $152 \pm 4^{\ddagger}$ \\
\hline \multicolumn{5}{|l|}{ Urine volume (ml/24 h) } \\
\hline Week 3 & $13.5 \pm 0.6$ & $13.8 \pm 1.1$ & $31.8 \pm 1.8^{\star}$ & $31.8 \pm 2.6^{*}$ \\
\hline Week 12 & $25.2 \pm 1.7$ & $25.8 \pm 1.8$ & $53.3 \pm 3.8^{\star}$ & $49.3 \pm 3.9 *$ \\
\hline \multicolumn{5}{|l|}{ Renal tissue weight (g) } \\
\hline To body weight (g/kg) & $6.4 \pm 0.2$ & $6.0 \pm 0.1$ & $5.2 \pm 0.3^{*}$ & $5.0 \pm 0.3^{\star}$ \\
\hline Right kidney (g) & $1.4 \pm 0.1$ & $1.2 \pm 0.1$ & removed & removed \\
\hline Left kidney (g) & $1.4 \pm 0.1$ & $1.2 \pm 0.1$ & $2.3 \pm 0.1^{*}$ & $2.1 \pm 0.1^{\star}$ \\
\hline \multicolumn{5}{|c|}{ Laboratory determinations at week 12} \\
\hline Uric acid $(\mu \mathrm{mol} / \mathrm{l})$ & $36 \pm 11$ & $117 \pm 21^{\star}$ & $63 \pm 19$ & $152 \pm 19 *^{\dagger}$ \\
\hline Creatinine $(\mu \mathrm{mol} / \mathrm{l})$ & $40 \pm 5$ & $49 \pm 3$ & $82 \pm 3^{*}$ & $83 \pm 8 *$ \\
\hline Creatinine clearance $(\mathrm{ml} / \mathrm{min})$ & $2.9 \pm 0.4$ & $2.0 \pm 0.2^{\star}$ & $1.2 \pm 0.1^{*}$ & $1.2 \pm 0.1^{*}$ \\
\hline \multirow[t]{2}{*}{ Renin activity (ng/ml/h) } & 27.3 & 31.2 & 2.0 & 5.0 \\
\hline & $(22.8-30.9)$ & $(27.8-41.4)$ & $(0.3-3.1)^{\star}$ & $(2.7-9.9) *$ \\
\hline Phosphate (mmol/l) & $1.4 \pm 0.1$ & $1.5 \pm 0.1$ & $1.9 \pm 0.2^{\ddagger}$ & $1.9 \pm 0.2^{\ddagger}$ \\
\hline Calcium (mmol/l) & $2.42 \pm 0.02$ & $2.36 \pm 0.02$ & $2.42 \pm 0.04$ & $2.42 \pm 0.02$ \\
\hline Sodium (mmol/l) & $136.5 \pm 0.5$ & $137.3 \pm 0.6$ & $136.7 \pm 0.9$ & $137.0 \pm 0.5$ \\
\hline
\end{tabular}

Values are mean \pm SEM or median (25th -75 th percentile), $* P<0.05$ compared with the Sham group, ${ }^{\dagger} P<0.05$ compared with the NX group, ${ }^{\#} \mathrm{P}<0.05$ oxonic acid treated groups versus untreated groups (two-way ANOVA), ${ }^{\ddagger} P<0.05$ NX groups compared with the Sham groups (two-way ANOVA). NX, 5/6 nephrectomized rat; Sham, sham-operated rat; Oxo, $2.0 \%$ oxonic acid diet. 


\begin{tabular}{|c|c|c|c|c|}
\hline & $\begin{array}{l}\text { Sham } \\
(n=9-12)\end{array}$ & $\begin{array}{l}\text { Sham + Oxo } \\
(n=12)\end{array}$ & $\begin{array}{l}N X \\
(n=11-12)\end{array}$ & $\begin{array}{l}N X+0 \times 0 \\
(n=12)\end{array}$ \\
\hline Potassium (mmol/l) & $4.1 \pm 0.1$ & $3.8 \pm 0.1$ & $4.3 \pm 0.2$ & $4.4 \pm 0.2$ \\
\hline \multicolumn{5}{|l|}{ copies (x $10^{4} /$ ng total RNA) } \\
\hline \multicolumn{5}{|c|}{$\begin{array}{l}\text { Values are mean } \pm \text { SEM or median ( } 25 \text { th }-75 \text { th percentile), }{ }^{*} P<0.05 \text { compared with the Sham group, } \\
{ }^{+} P<0.05 \text { compared with the NX group, }{ }^{\#} \mathrm{P}<0.05 \text { oxonic acid treated groups versus untreated groups } \\
\text { (two-way ANOVA), }{ }^{\ddagger} P<0.05 \mathrm{NX} \text { groups compared with the Sham groups (two-way ANOVA). NX, } 5 / 6 \\
\text { nephrectomized rat; Sham, sham-operated rat; Oxo, } 2.0 \% \text { oxonic acid diet. }\end{array}$} \\
\hline
\end{tabular}

\subsection{Laboratory determinations}

The $2 \%$ Oxo diet elevated plasma UA levels $~ 2.5$ to 3 -fold in the NX and Sham rats, respectively (Table 1). Plasma creatinine was elevated by $35-42 \mu \mathrm{mol} / \mathrm{I}$ and creatinine clearance decreased by $\sim 60 \%$ in the NX rats, while hyperuricemia reduced creatinine clearance by $~ 30 \%$ in the Sham rats. Suppressed plasma renin activity and moderate phosphate retention was observed in both NX groups, whereas the plasma concentrations of calcium, sodium and potassium were corresponding in all groups (Table 1). The 24hour urinary protein excretion was clearly increased in both NX groups (Fig. 1A).

\subsection{Renal histology}

The indices of arteriosclerosis (Fig. 1B), glomerulosclerosis (Fig. 1C), and tubulointerstitial damage (Fig. 1D) were increased in the NX group, while all these indices of renal damage were alleviated in the NX-Oxo when compared with the NX group. The glomerulosclerosis score also significantly correlated with the 24-hour urinary protein excretion (Fig. 1E).

Staining of SMA was evaluated in an attempt to identify afferent arterioles, as afferent arteriolopathy has been previously reported in a setting where rats were put on $2 \%$ Oxo diet immediately after surgical $5 / 6$ NX operation and followed for 6 weeks $(6,17)$. We observed a clear SMA staining of the arteries that were adjacent to the glomeruli (Fig. 1F). However, we could not reliably differentiate the afferent arterioles from the efferent arterioles with the present techniques. Therefore, further analysis of the preglomerular arterioles was not performed.

\subsection{Markers of inflammation, oxidative stress, and collagen I in the kidney}

Already 3 weeks after the NX operation when the $2 \%$ Oxo diet commenced (study week 3 ), the 24 hour excretion of 11-epi-prostaglandin- $\mathrm{F}_{2 \mathrm{a}}$ to the urine was $\sim 1.6$-fold higher in the NX groups than in the Sham groups (Fig. 2A). At study week 12, the 24-hour urinary 11-epi-prostaglandin- $F_{2 a}$ excretion was 2.3-fold 
higher in the NX group than in the Sham group, whereas the excretion was reduced by more than $70 \%$ in both groups ingesting the $2 \%$ Oxo-diet (Fig. 2B).

Kidney tissue HO-1 mRNA content was higher in both NX groups than in the Sham groups (Fig. 2C). The number of mast cells in the kidney tissue was elevated following subtotal nephrectomy, whilst the mast number was significantly lower after the Oxo diet in the remnant kidney rats (Fig. 2D). A significant direct correlation between the kidney mast cell count and 24-hour urinary 11 epi-prostaglandin $-\mathrm{F}_{2 a}$ excretion $\left(r_{S}=0.415, p=0.003\right)$ was also observed.

A clear staining of both glomerular and tubulointerstitial COX-2 was observed in the kidney sections (Figs. 3A and 3B). Tubulointerstitial COX-2 score was lower in the Sham + Oxo group than in the Sham group, and in the NX+ Oxo than group than in the NX group (Fig. $3 \mathrm{C}$ ). The COX-2 staining was abundant in tubuli with thick epithelium corresponding to the ascending limb of the loop of Henle (28). In the glomeruli, the number of COX2 positive cells was lower in the Sham + Oxo group than in the Sham group, while lowest COX2 positive cell numbers were observed in the glomeruli of the NX groups (Fig. 3D). The present surgical remnant kidney model was associated with increased kidney tissue collagen I mRNA expression (two-way ANOVA $p=0.022$ ), but collagen I mRNA expression was not influenced by the Oxo diet (Table 1).

\section{Discussion}

Here we examined the effects of $2 \%$ Oxo diet induced hyperuricemia on kidney morphology, inflammation, and markers of oxidative stress in a low-renin model of experimental CRI. Previously, we found that $2 \%$ Oxo diet elevated plasma renin and aldosterone, but in parallel improved NO-mediated vasorelaxation in the carotid artery, and reduced oxidative stress in vivo in remnant kidney rats $(9,20)$. The current results showed that moderately elevated plasma UA level was associated with favorable changes in kidney histology and reduced markers of inflammation in NX rats.

During $2 \%$ Oxo feeding a moderate rise in circulating UA levels is achieved due to inhibition of the hepatic enzyme uricase that metabolizes UA to its final end-product allantoin (16). Subsequently, plasma UA levels are elevated to concentrations that are closer to those observed in humans. The present 2.5-3-fold elevations of UA levels induced by the Oxo diet well correspond to previous findings $(6,16,17,19)$. Pharmacological lowering of serum UA levels was not included in the present protocol, as several studies have shown that the xanthine oxidase (XO) inhibitors allopurinol and febuxostat effectively prevent the effects of Oxo diet in rats $(5,16,17,19)$. Of note, the therapeutic effects of $X O$ inhibitors have not been solely related to reduced UA concentrations, but also to the anti-oxidative and anti-inflammatory properties of these compounds (29). The $5 / 6 \mathrm{NX}$ model has also been characterized by reduced tissue XO activity and a compensatory increase in intestinal UA excretion (30). These mechanisms may explain why plasma UA was not significantly higher in the NX rats on the normal diet than in Sham rats on the normal diet. 
The present renal insufficiency 12 weeks after the NX operation was documented by elevated plasma creatinine, reduced creatinine clearance, hyperphosphatemia, and increased urinary protein excretion (19). The histology showed increased indices of arteriosclerosis, glomerulosclerosis, and tubulointerstitial damage in the NX group. The reliability of the histological findings is supported by the good correlation between the glomerulosclerosis score and the amount of 24-hour urinary protein excretion. The hypertrophy of the remnant kidney can be attributed to compensatory tissue growth in an attempt to compensate for the reduced renal function $(31,32)$. The NX rats presented with low plasma renin activity probably due to the associated volume load, corresponding to previous findings in rats subjected to surgical renal ablation (20). Systolic BP was only modestly elevated 12 weeks after renal ablation, as more marked hypertension is known to develop only later in the course impaired renal function in this surgical low-renin model $(33,34)$.

Previously, the harmful effects of high UA concentrations in renal tissue have been attributed to the deposition of non-soluble monosodium urate crystals in renal tubules (gouty nephropathy) (35). Intracellularly UA may also mediate biological effects that may play a role in the development of subclinical "non-gouty" types of renal and cardiovascular disease (35). Excess generation of reactive oxygen species (ROS) has been suggested to play a central role in the UA-induced renal disease (5). The interaction between UA and ROS is complex, as the synthesis of UA from its purine and pyrimidine nucleotide precursors is catalyzed by two xanthine oxidoreductase enzymes: xanthine dehydrogenase and XO. In ischemic states such as CRI, the latter is the predominant catalyzer creating ROS, mainly superoxide anion, as a by-product of the UA synthesis. In cell cultures, UA can inhibit renal production of NO synthase, a catalyzing enzyme in NO generation (16). The reaction between ROS and NO may result in renal NO-depletion and afferent artery vasoconstriction, which is an essential step in renal fibrosis (17). On the other hand, the antioxidant properties of UA are widely accepted. By scavenging superoxide anions UA can prevent it from reacting with NO and thus inhibit the formation of the toxic peroxynitrite $(10,36)$. Also, the reaction of UA with peroxynitrite yields a nitrated UA derivate, which has vasodilatory effects (37). Finally, UA can also counter oxidant-induced renal injury by preventing the inactivation of extracellular superoxide dismutase, an enzyme that provides tissue protection by catalyzing the dismutation of superoxide radical into oxygen and hydrogen peroxide $(38,39)$.

We found that the number of mast cells was elevated in remnant kidneys, while mast cell quantity was reduced following the Oxo diet. Kidney mast cell density is known to correlate with the severity of renal disease (40). Various etiologies, such as several forms of nephropathies and renovascular ischemia that cause glomerular damage and interstitial fibrosis, are associated with mast cell abundance in the kidney (40). Mast cells can aggravate tissue damage and fibrosis by recruiting leukocytes, profibrogenic cytokines, proteases, and growth factors, and also by directly stimulating collagen synthesis (40). In an experimental rat model, a close association between mast cell density and oxidative stress in the kidney, as indicated by superoxide anion generation, was previously reported (41). We also assessed mast cell activity by measuring the quantity of mast cellderived prostaglandin $\mathrm{D}_{2}\left(\mathrm{PGD}_{2}\right)$ metabolite, 11-epiprostaglandin- $F_{2 a}$, in the urine $(27,42)$. Due to the long half-life and stability, 11-epi-prostaglandin- $F_{2 a}$ is a 
convenient way to evaluate mast cell activity in vivo $(27,42,43)$. The present findings of kidney mast cell density and urinary 24-hour 11-epi-prostaglandin $-\mathrm{F}_{2 a}$ excretion were congruent, and a direct correlation between these variables was observed. Possible explanations to the reduced mast cell infiltration and activity in the renal tissue of hyperuricemic NX rats are decreased amounts of ROS and increased NO bioavailability (9), as both of these factors can reduce tissue inflammation and inhibit mast cell degranulation $(41,44)$.

Whether the actions of UA are detrimental or beneficial may depend on the distribution of UA between the intra- and extracellular compartments (35). Extracellularly the antioxidant properties predominate, whereas intracellularly UA may be a pro-oxidant (35). For instance, the free radical scavenging capability of plasma UA appears to have favorable effects on kidney tissue in CRI (45). In contrast, the blockade of UA entry into the renal tubular cells by the organic anion transporter inhibitor probenecid prevented epithelial-to-mesenchymal transition, an event contributing to progressive tubular fibrosis (46). We found that kidney tissue H0-1 mRNA content was higher in both NX groups than in the Sham groups but did not differ between the Sham and Sham + Oxo groups, or between the NX and NX+ Oxo groups. These findings support the view that the present Oxo diet did not cause oxidative stress even at the cellular level in vivo. Tissue HO-1 content serves as an index of oxidant stress in humans and in animal models of renal disease (47-49). By converting cell toxic heme to biliverdin in a reaction that liberates carbon monoxide (CO) and iron, $\mathrm{HO}-1$ counteracts oxidant burden. Inactivation of heme by HO-1 prevents it from inducing lipid peroxidation, and ROS and hydrogen peroxide generation in tubular epithelial cells, while the reaction by-products biliverdin and $\mathrm{CO}$ in low concentrations possess antioxidant and vasodilatory effects (50). In CRI, biliverdin ja CO can even help to preserve normal glomerular filtration rate and sodium handling by suppressing tubule-glomerular feedback and afferent arteriolar vasoconstriction (48).

In the mammalian kidneys, COX-2 has been mainly localized to the macula densa, cortical thick ascending limb, and medullary interstitial cells (28). Increased juxtaglomerular renin and preglomerular arterial COX-2 production have been suggested to contribute to smooth muscle cell proliferation and renal arteriolar obliteration in experimental hyperuricemia (6). However, in the present study the number of glomerular COX-2 positive cells was reduced by $2 \%$ Oxo feeding in the Sham rats and was equally further reduced in both NX groups. The explanation for the reduced number of glomerular COX-2 positive cells in both NX groups remains unknown, but may be related to the glomerular hypertrophy and hyperfiltration caused by surgical subtotal nephrectomy $(20,32)$. We found that experimental hyperuricemia suppressed tubulointerstitial COX2 protein staining. These findings suggest reduced COX-2 derived inflammatory influences in the kidneys after the $2 \%$ Oxo diet. Lower kidney tissue COX-2 content is well in line with the beneficial effects of experimental hyperuricemia on renal histology in the NX rats. Of note, in addition to mast cells, 11-epi-prostaglandin- $\mathrm{F}_{2 a}$ can also originate from prostanoids synthetized via COX-2.

Therefore, reduced 11-epi-prostaglandin- $\mathrm{F}_{2 a}$ excretion may also reflect reduced total COX-2 content in the kidneys of the hyperuricemic rats (51).

Immunohistochemical staining of SMA was done in order to examine the renal preglomerular arterioles, as thickening of the afferent arterioles has been suggested to trigger UA-induced renal fibrosis (17). The 
renal arterioles were clearly identified adjacent to glomeruli, but we were unable to reliably differentiate the afferent from the efferent renal arterioles. Therefore, the present results are inconclusive with respect to preglomerular small artery structure. Increased interstitial collagen deposition has been suggested to mediate UA-mediated renal fibrosis (16). In the present study, collagen I mRNA expression was elevated in CRI but was not influenced by moderate hyperuricemia.

The causal role of UA in the progression of renal disease has been questioned by Mendelian randomization studies $(52,53)$. A recent review concluded that the causal association of UA with a range of health outcomes is evident only in gout and nephrolithiasis (54). In hemodialysis patients, lower UA levels were independently associated with higher all-cause and cardiovascular mortality (55), while in patients with end-stage renal disease not receiving dialysis or receiving peritoneal dialysis, higher serum UA associated with higher mortality (53). There is also evidence that subjects genetically predisposed to hypouricemia present with an elevated risk for renal disease (56). The optimal range of circulating UA levels in various health conditions warrants further research.

\section{Conclusions}

Consistent with previous reports, the surgical remnant kidney low-renin model of CRI was characterized by renal scarring and increased proteinuria. These pathological alterations were related to increased renal inflammation, fibrosis, and oxidative stress, as indicated by increased mast cell infiltration and activation, elevated collagen I mRNA, and elevated HO-1 mRNA in the kidneys of the NX rats. Nine weeks of 2\% Oxo diet increased plasma UA concentrations and improved renal histology with a parallel reduction in local and urinary markers of inflammation in the remnant kidney rats. These findings indicate that elevated UA levels, which increase the antioxidant capacity in plasma (9), do not always cause histological and functional impairment of the kidneys.

\section{Abbreviations}

RT-PCR, real-time PCR; mRNA, messenger RNA; COX-2, cyclooxygenase-2; CRI, chronic renal insufficiency; UA, uric acid; Oxo, oxonic acid; RAS, renin-angiotensin system; NX, nephrectomy; NO, nitric oxide; BP, blood pressure; PAS, periodic acid Schiff; SMA, smooth muscle cell alpha-actin; HO-1, heme oxygenase-1; ANOVA, analysis of variance; SEM, standard errors of the mean; $X O$, xanthine oxidase; ROS, reactive oxygen species; PGD2, prostaglandin D2; CO, carbon monoxide

\section{Declarations}

\section{Ethics approval}

The study was approved by the Animal Experimentation Committee of the University of Tampere, and the Provincial Government of Western Finland Department of Social Affairs and Health, Finland (decision 
LSLH-2003-9718/Ym-23) and conforms to the Guiding Principles for Research Involving Animals. All methods are reported in accordance with ARRIVE guidelines (https://arriveguidelines.org).

\section{Consent for publication}

Not applicable.

\section{Availability of data and materials}

All data generated or analyzed during this study are included in this published article.

\section{Competing interest}

The authors declare that they have no competing interests.

\section{Funding}

This work was supported by the Finnish Foundation of Cardiovascular Research, Pirkanmaa Regional Fund of the Finnish Cultural Foundation, and Competitive State Research Financing of the Expert Responsibility Area of Tampere University Hospital (grant number 9E054).

\section{Authors' contributions}

Venla Kurra: Investigation, formal analysis, writing - original draft. Arttu Eräranta: Methodology, investigation, visualization. Timo Paavonen: Methodology, resources. Teemu Honkanen: Methodology, investigation. Juhani Myllymäki: Investigation. Asko Riutta: Methodology, investigation. Ilkka Tikkanen: Methodology, resources. Päivi Lakkisto: Methodology, formal analysis. Jukka Mustonen: Conceptualization, supervision. Ilkka Pörsti: Conceptualization, methodology, resources, writing reviewing and editing, supervision, administration, funding acquisition. All authors contributed to the study design, analysis, and interpretation of data, and revising the article critically and they approved the final version of the manuscript.

\section{Acknowledgements}

The invaluable help of Peeter Kööbi, MD, PhD, and Tuija Vehmas, MS, in the course of the experiments is sincerely acknowledged.

\section{References}


1. Murea M. Advanced kidney failure and hyperuricemia. Adv Chronic Kidney Dis. 2012 Nov;19(6):41924.

2. Johnson RJ, Bakris GL, Borghi C, Chonchol MB, Feldman D, Lanaspa MA, et al. Hyperuricemia, acute and chronic kidney disease, hypertension, and cardiovascular disease: report of a scientific workshop organized by the national kidney foundation. Am J Kidney Dis. 2018 Jun 1;71(6):851-65.

3. Johnson RJ, Herrera-Acosta J, Schreiner GF, Rodriguez-Iturbe B. Subtle acquired renal injury as a mechanism of salt-sensitive hypertension. N Engl J Med. 2002 Mar 21;346(12):913-23.

4. Khosla UM, Zharikov S, Finch JL, Nakagawa T, Roncal C, Mu W, et al. Hyperuricemia induces endothelial dysfunction. Kidney Int. 2005 May;67(5):1739-42.

5. Sánchez-Lozada LG, Soto V, Tapia E, Avila-Casado C, Sautin YY, Nakagawa T, et al. Role of oxidative stress in the renal abnormalities induced by experimental hyperuricemia. Am J Physiol - Ren Physiol. 2008 Oct;295(4):F1134-41.

6. Kang D-H. A Role for Uric Acid in the Progression of Renal Disease. J Am Soc Nephrol. 2002 Dec 1;13(12):2888-97.

7. Fan S, Zhang P, Wang AY, Wang X, Wang L, Li G, et al. Hyperuricemia and its related histopathological features on renal biopsy. BMC Nephrol [Internet]. 2019 Mar 18 [cited 2020 May 21];20. Available from: https://www.ncbi.nlm.nih.gov/pmc/articles/PMC6423852/

8. Myllymaki J, Honkanen T, Syrjanen J, Helin H, Rantala I, Pasternack A, et al.Uric acid correlates with the severity of histopathological parameters in IgA nephropathy. Nephrol Dial Transpl. 2005 Jan;20(1):89-95.

9. Kurra V, Eraranta A, Jolma P, Vehmas TI, Riutta A, Moilanen E, et al. Hyperuricemia, oxidative stress, and carotid artery tone in experimental renal insufficiency. Am J Hypertens. 2009 Sep 1;22(9):96470 .

10. Ames BN, Cathcart R, Schwiers E, Hochstein P. Uric acid provides an antioxidant defense in humans against oxidant- and radical-caused aging and cancer: a hypothesis. Proc Natl Acad Sci U S A. 1981 Nov;78(11):6858-62.

11. Durante P, Romero F, Pérez M, Chávez M, Parra G. Effect of uric acid on nephrotoxicity induced by mercuric chloride in rats. Toxicol Ind Health. 2010 Apr 1;26(3):163-74.

12. W. Waring S, J. Webb D, R. J. Maxwell S. Systemic uric acid administration increases serum antioxidant capacity in healthy volunteers. J Cardiovasc Pharmacol. 2001 Sep;38(3):365-71.

13. Patschan D, Patschan S, Gobe GG, Chintala S, Goligorsky MS. Uric acid heralds ischemic tissue injury to mobilize endothelial progenitor cells. J Am Soc Nephrol. 2007 May;18(5):1516-24.

14. Wang Y-F, Li J-X, Sun X-S, Lai R, Sheng W-L. High serum uric acid levels are a protective factor against unfavourable neurological functional outcome in patients with ischaemic stroke. $\mathrm{J}$ Int Med Res. 2018 May;46(5):1826-38.

15. Arévalo-Lorido JC, Carretero-Gómez J, Robles NR. Serum uric acid levels and outcome during admission in acute ischaemic stroke, depending on renal function. Int J Neurosci. 2018 Oct 3;128(10):906-12. 
16. Mazzali M, Hughes J, Kim Y-G, Jefferson JA, Kang D-H, Gordon KL, et al. Elevated uric acid increases blood pressure in the rat by a novel crystal-independent mechanism.Hypertension. 2001 Nov;38(5):1101-6.

17. Mazzali M, Kanellis J, Han L, Feng L, Xia Y-Y, Chen Q, et al.Hyperuricemia induces a primary renal arteriolopathy in rats by a blood pressure-independent mechanism. Am J Physiol-Ren Physiol. 2002 Jun 1;282(6):F991-7.

18. Nakagawa T, Mazzali M, Kang D-H, Kanellis J, Watanabe S, Sanchez-Lozada LG, et al. Hyperuricemia causes glomerular hypertrophy in the rat. Am J Nephrol.2003;23(1):2-7.

19. Sanchez-Lozada LG, Tapia E, Santamaria J, Avila-Casado C, Soto V, Nepomuceno T, et al.Mild hyperuricemia induces vasoconstriction and maintains glomerular hypertension in normal and remnant kidney rats.Kidney Int. 2005 Jan;67(1):237-47.

20. Eräranta A, Kurra V, Tahvanainen AM, Vehmas TI, Kööbi P, Lakkisto P, et al.Oxonic acid-induced hyperuricemia elevates plasma aldosterone in experimental renal insufficiency.J Hypertens. 2008 Aug;26(8):1661-8.

21. Jolma P, Koobi P, Kalliovalkama J, Saha H, Fan M, Jokihaara J, et al.Treatment of secondary hyperparathyroidism by high calcium diet is associated with enhanced resistance artery relaxation in experimental renal failure. Nephrol Dial Transplant. 2003 Dec 1;18(12):2560-9.

22. Kööbi P, Kalliovalkama J, Jolma P, Rysä J, Ruskoaho H, Vuolteenaho O, et al. At ${ }_{1}$ receptor blockade improves vasorelaxation in experimental renal failure. Hypertension. 2003 Jun;41(6):1364-71.

23. Prætorius E, Poulsen H. Enzymatic determination of uric acid with detailed directions. Scand J Clin Lab Invest. 1953 Jan 1;5(3):273-80.

24. Schwarz U, Amann K, Orth SR, Simonaviciene A, Wessels S, Ritz E. Effect of 1,25(OH)2 vitamin D3 on glomerulosclerosis in subtotally nephrectomized rats. Kidney Int. 1998 Jun;53(6):1696-705.

25. Racusen LC, Solez K, Colvin RB, Bonsib SM, Castro MC, Cavallo T, et al. The Banff 97 working classification of renal allograft pathology. Kidney Int. 1999 Feb 1;55(2):713-23.

26. Rantanen T, Udd M, Honkanen T, Miettinen P, Kärjä V, Rantanen L, et al.Effect of omeprazole dose, nonsteroidal anti-inflammatory agents, and smoking on repair mechanisms in acute peptic ulcer bleeding. Dig Dis Sci. 2014 Nov 1;59(11):2666-74.

27. Mucha I, Riutta A. Determination of 9 a, $11 \beta$-prostaglandin F2 in human urine. Combination of solidphase extraction and radioimmunoassay. Prostaglandins Leukot Essent Fatty Acids. 2001 Nov $1 ; 65(5): 271-80$.

28. Harris RC, Breyer MD. Physiological regulation of cyclooxygenase-2 in the kidney. Am J Physiol-Ren Physiol. 2001 Jul 1;281(1):F1-11.

29. George J, Carr E, Davies J, Belch JJF, Struthers A. High-dose allopurinol improves endothelial function by profoundly reducing vascular oxidative stress and not by lowering uric acid. Circulation. 2006 Dec 5;114(23):2508-16. 
30. Vaziri ND, Freel RW, Hatch M. Effect of chronic experimental renal insufficiency on urate metabolism. J Am Soc Nephrol. 1995 Oct 1;6(4):1313-7.

31. Hayslett JP. Functional adaptation to reduction in renal mass. Physiol Rev. 1979 Jan 1;59(1):13764.

32. Amann K, Rump LC, Simonaviciene A, Oberhauser V, Wessels S, Orth SR, et al. Effects of low dose sympathetic inhibition on glomerulosclerosis and albuminuria in subtotally nephrectomized rats.J Am Soc Nephrol. 2000;11:1469-78.

33. Kööbi P, Vehmas TI, Jolma P, Kalliovalkama J, Fan M, Niemelä O, et al.High-calcium vs highphosphate intake and small artery tone in advanced experimental renal insufficiency. Nephrol Dial Transplant. 2006 Oct 1;21(10):2754-61.

34. Eräranta A, Riutta A, Fan M, Koskela J, Tikkanen I, Lakkisto P, et al.Dietary phosphate binding and loading alter kidney angiotensin-converting enzyme mRNA and protein content in 5/6 nephrectomized rats. Am J Nephrol. 2012;35(5):401-8.

35. Sanchez-Lozada LG, Rodriguez-Iturbe B, Kelley EE, Nakagawa T, Madero M, Feig DI, et al. Uric acid and hypertension: an update with recommendations. Am J Hypertens [Internet]. [cited 2020 May 21]; Available from: https://academic.oup.com/ajh/advance-article/doi/10.1093/ajh/hpaa044/5807974

36. Glantzounis GK, Tsimoyiannis EC, Galaris AMK and DA. Uric acid and oxidative stress. Curr Pharm Des [Internet]. 2005 Nov 30 [cited 2020 May 22];11(32):4145-51. Available from: http://www.eurekaselect.com/60508/article

37. Skinner KA, White CR, Patel R, Tan S, Barnes S, Kirk M, et al. Nitrosation of uric acid by peroxynitrite: formation of a vasoactive nitric oxide donor. J Biol Chem. 1998 Sep 18;273(38):24491-7.

38. Hink HU, Santanam N, Dikalov S, McCann L, Nguyen AD, Parthasarathy S, et al. Peroxidase properties of extracellular superoxide dismutase: role of uric acid in modulating in vivo activity. Arterioscler Thromb Vasc Biol. 2002 Sep;22(9):1402-8.

39. Tan RJ, Zhou D, Xiao L, Zhou L, Li Y, Bastacky SI, et al. Extracellular superoxide dismutase protects against proteinuric kidney disease. J Am Soc Nephrol. 2015 Oct;26(10):2447-59.

40. Madjene LC, Pons M, Danelli L, Claver J, Ali L, Madera-Salcedo IK, et al. Mast cells in renal inflammation and fibrosis: Lessons learnt from animal studies. Mol Immunol. 2015 Jan 1;63(1):8693.

41. Reena, Kaur T, Kaur A, Singh M, Buttar HS, Pathak D, et al. Mast cell stabilizers obviate high fat dietinduced renal dysfunction in rats. Eur J Pharmacol. 2016 Apr 15;777:96-103.

42. Pugliese G, Spokas EG, Marcinkiewicz E, Wong PY. Hepatic transformation of prostaglandin D2 to a new prostanoid, 9 alpha, 11 beta-prostaglandin F2, that inhibits platelet aggregation and constricts blood vessels. J Biol Chem. 1985 Nov 25;260(27):14621-5.

43. O'Sullivan S, Dahlén B, Dahlén S-E, Kumlin M. Increased urinary excretion of the prostaglandin D2 metabolite $9 a, 11 \beta$-prostaglandin F2 after aspirin challenge supports mast cell activation in aspirininduced airway obstruction. J Allergy Clin Immunol. 1996 Aug 1;98(2):421-32. 
44. Coleman JW. Nitric oxide: a regulator of mast cell activation and mast cell-mediated inflammation. Clin Exp Immunol. 2002 Jul;129(1):4-10.

45. Murea M, Tucker BM. The physiology of uric acid and the impact of end-stage kidney disease and dialysis. Semin Dial. 2019;32(1):47-57.

46. Ryu E-S, Kim MJ, Shin H-S, Jang Y-H, Choi HS, Jo I, et al. Uric acid-induced phenotypic transition of renal tubular cells as a novel mechanism of chronic kidney disease. Am J Physiol-Ren Physiol. 2013 Mar 1;304(5):F471-80.

47. Morimoto K, Ohta K, Yachie A, Yang Y, Shimizu M, Goto C, et al. Cytoprotective role of heme oxygenase (HO)-1 in human kidney with various renal diseases. Kidney Int. 2001 Nov;60(5):1858-66.

48. Nath KA. Heme oxygenase-1: A provenance for cytoprotective pathways in the kidney and other tissues. Kidney Int. 2006 Aug;70(3):432-43.

49. Diwan V, Mistry A, Gobe G, Brown L. Adenine-induced chronic kidney and cardiovascular damage in rats. J Pharmacol Toxicol Methods. 2013 Sep 1;68(2):197-207.

50. Ferenbach DA, Kluth DC, Hughes J. Hemeoxygenase-1 and Renal Ischaemia-Reperfusion Injury. Nephron Exp Nephrol. 2010;115(3):e33-7.

51. Vainio M, Riutta A, Koivisto A-M, Mäenpää J. 9a,11ß-prostaglandin F2 in pregnancies at high risk for hypertensive disorders of pregnancy, and the effect of acetylsalicylic acid. Prostaglandins Leukot Essent Fatty Acids. 2003 Jul 1;69(1):79-83.

52. Hughes K, Flynn T, Zoysa J de, Dalbeth N, Merriman TR. Mendelian randomization analysis associates increased serum urate, due to genetic variation in uric acid transporters, with improved renal function. Kidney Int. 2014 Jan 1;85(2):344-51.

53. Ahola AJ, Sandholm N, Forsblom C, Harjutsalo V, Dahlström E, Groop P-H. The serum uric acid concentration is not causally linked to diabetic nephropathy in type 1 diabetes. Kidney Int. 2017 May 1;91(5):1178-85.

54. Li X, Meng X, Timofeeva M, Tzoulaki I, Tsilidis KK, loannidis PA, et al. Serum uric acid levels and multiple health outcomes: umbrella review of evidence from observational studies, randomised controlled trials, and Mendelian randomisation studies. The BMJ [Internet]. 2017 Jun 7 [cited 2020 May 11];357. Available from: https://www.ncbi.nlm.nih.gov/pmc/articles/PMC5461476/

55. Sugano N, Maruyama Y, Kidoguchi S, Ohno I, Wada A, Shigematsu T, et al. Effect of hyperuricemia and treatment for hyperuricemia in Japanese hemodialysis patients: A cohort study. PLoS ONE [Internet]. 2019 Jun 6 [cited 2020 May 21];14(6). Available from: https://www.ncbi.nlm.nih.gov/pmc/articles/PMC6553731/

56. Dissanayake LV, Spires DR, Palygin O, Staruschenko A. Effects of uric acid dysregulation on the kidney. Am J Physiol-Ren Physiol. 2020 Mar 30;318(5):F1252-7.

\section{Figures}



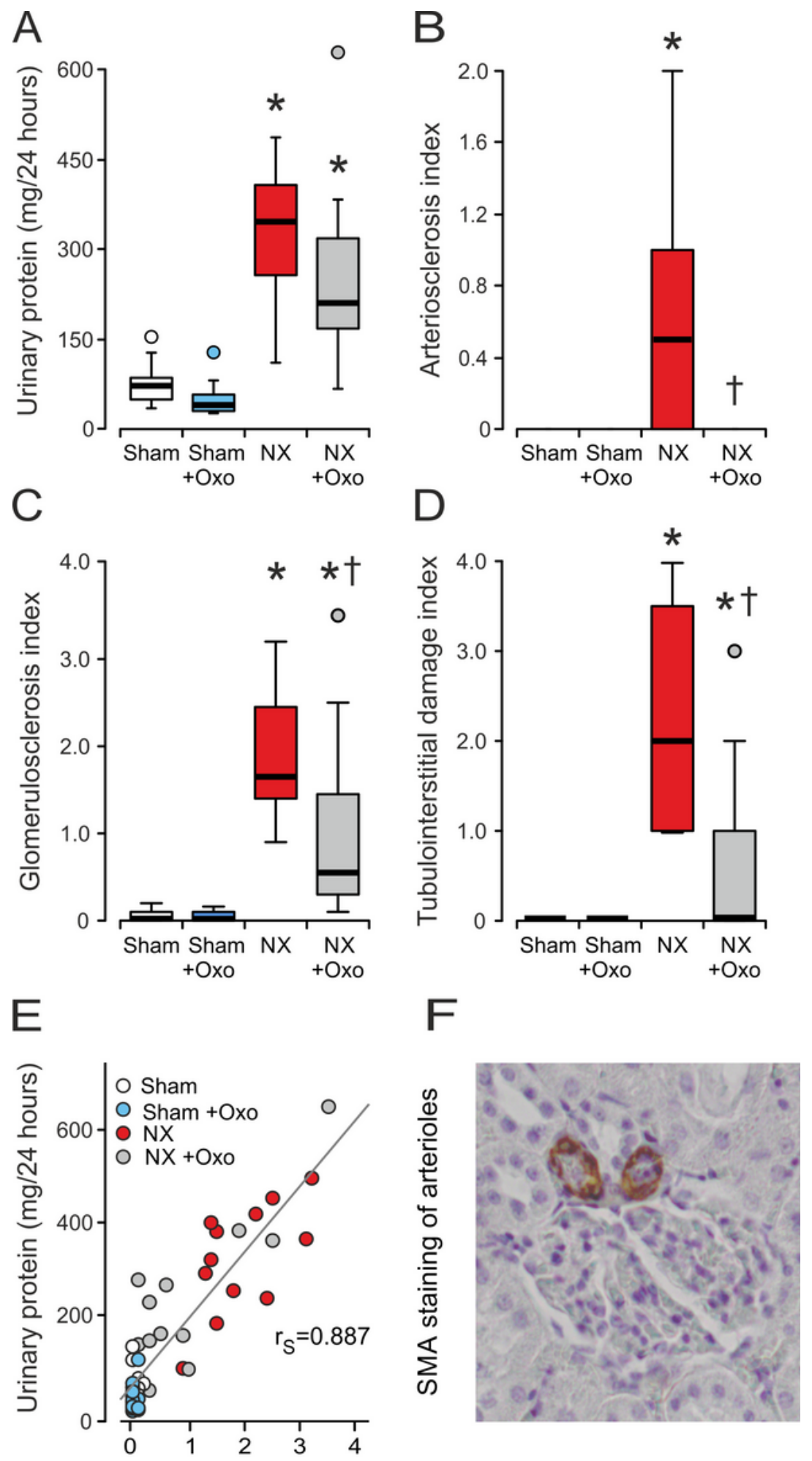

Glomerulosclerosis score

\section{Figure 1}

Urinary protein excretion during study week 12 (A), kidney arteriosclerosis index (B), glomerulosclerosis index (C), tubulointerstitial damage index (D), correlation between urinary protein excretion and glomerulosclerosis score (E), and representative photomicrograph of smooth muscle actin (SMA) staining of the glomerular arterioles ( $F)$ in the study groups ( $n=11-12$ in each group). NX, 5/6 nephrectomized rat; Sham, sham-operated rat; Oxo, 2.0\% oxonic acid diet. Values are median (thick line), 
$25^{\text {th }}-75^{\text {th }}$ percentile (box), and range (whiskers), outliers depicted as small circles; ${ }^{*} P<0.05$ versus Sham, ${ }^{\dagger} P<0.05$ versus NX.
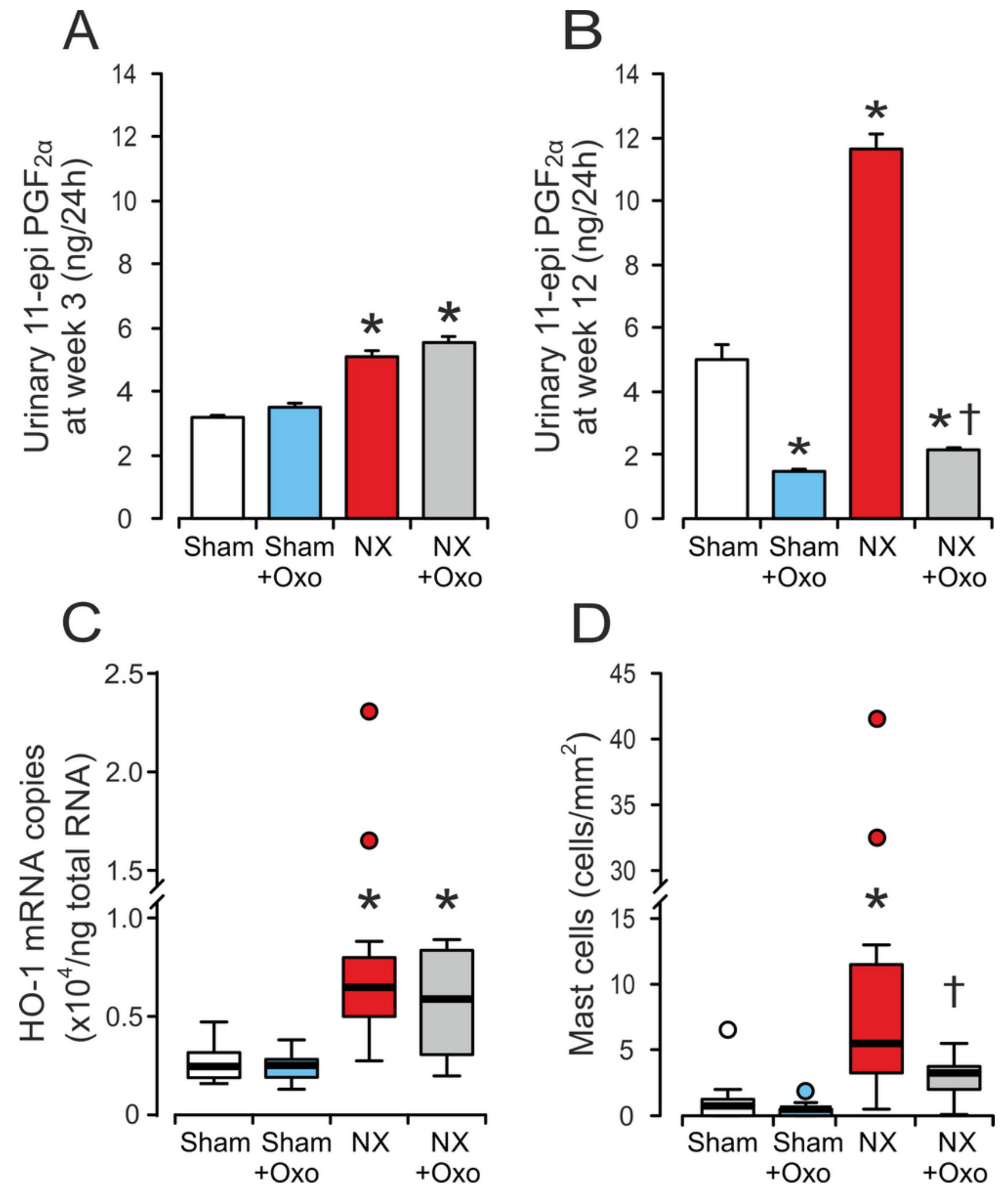

Figure 2 
The 24-hour urinary excretion of 11-epi-prostaglandin $\mathrm{F}_{2 a}$ during study week 3 (A) and study week 12 (B), kidney hemooxygenase-1 (HO-1) mRNA expression (C), and kidney mast cell content (D) in the study groups ( $n=12$ in each group). Groups as in Figure 1 ; mean $\pm S E M$, or median (thick line), $25^{\text {th }}-75^{\text {th }}$ percentile (box), and range (whiskers), outliers depicted as small circles; ${ }^{*} P<0.05$ versus Sham, ${ }^{\dagger} P<0.05$ versus NX.
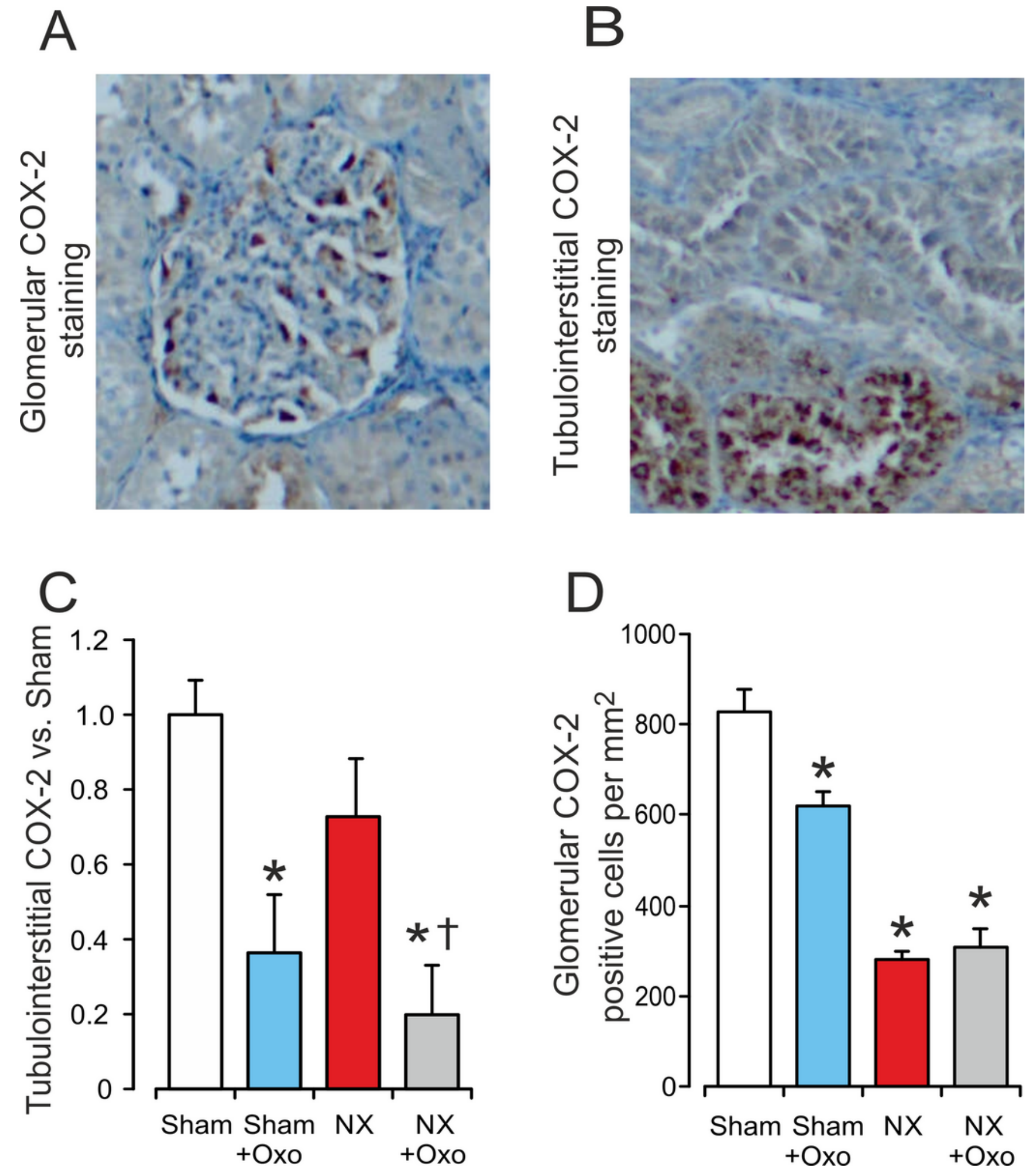


\section{Figure 3}

Representative photomicrographs of the immunohistochemical staining of glomerular $(A)$ and tubulointerstitial (B) cyclooxygenase-2 (COX-2), tubulointerstitial COX-2 score (C), number of COX-2 positive cells in the glomeruli (D) in the study groups ( $n=9-11$ in each group). Groups as in Figure 1 , values are mean \pm SEM; ${ }^{*} P<0.05$ versus Sham, ${ }^{\dagger} P<0.05$ versus NX. 